\title{
A GENERALIZATION OF A THEOREM OF NAGELL
}

\author{
YULU FENG, SHAOFANG HONG*,, XIAO JIANG AND QIUYU YIN \\ MATHEMATICAL COLLEGE, SICHUAN UNIVERSITY, CHENGDU 610064, P.R. CHINA \\ E-MAILS: YULUFENG17@126.COM (Y.L. FENG); SFHONG@SCU.EDU.CN, \\ S-F.HONG@TOM.COM, HONGSF02@YAHOO.COM (S.F.HONG); 422040631@QQ.COM \\ (X. JIANG); YINQIUYU26@126.COM (Q.Y. YIN)
}

\begin{abstract}
Let $n$ be a positive integer. In 1915, Theisinger proved that if $n \geq 2$, then the $n$-th harmonic sum $\sum_{k=1}^{n} \frac{1}{k}$ is not an integer. Let $a$ and $b$ be positive integers. In 1923, Nagell extended Theisinger's theorem by showing that the reciprocal sum $\sum_{k=1}^{n} \frac{1}{a+(k-1) b}$ is not an integer if $n \geq 2$. In 1946, Erdős and Niven proved a theorem of a similar nature that states that there is only a finite number of integers $n$ for which one or more of the elementary symmetric functions of $1,1 / 2, \ldots, 1 / n$ is an integer. In this paper, we present a generalization of Nagell's theorem. In fact, we show that for arbitrary $n$ positive integers $s_{1}, \ldots, s_{n}$ (not necessarily distinct and not necessarily monotonic), the following reciprocal power sum

$$
\sum_{k=1}^{n} \frac{1}{(a+(k-1) b)^{s_{k}}}
$$

is never an integer if $n \geq 2$. The proof of our result is analytic and $p$-adic in character.
\end{abstract}

\section{INTRODUCTION}

Let $\mathbb{Z}, \mathbb{Z}^{+}$and $\mathbb{Q}$ be the set of integers, the set of positive integers and the set of rational numbers, respectively. Let $n \in \mathbb{Z}^{+}$. More than one hundred years ago, Theisinger [7] proved that the $n$-th harmonic sum $1+\frac{1}{2}+\ldots+\frac{1}{n}$ is never an integer if $n>1$. Eight years later, Nagell [6] extended Theisinger's theorem from the sequence of positive integers to general arithmetic progressions by showing that if $a$ and $b$ are positive integers and $n \geq 2$, then the reciprocal sum $\sum_{i=0}^{n-1} \frac{1}{a+b i}$ is never an integer. Then Erdös and Niven 2] generalized Nagell's theorem by establishing a similar result on the integrality of the elementary symmetric functions of $\frac{1}{a}, \frac{1}{a+b}, \ldots, \frac{1}{a+(n-1) b}$. In the recent years, Erdős and Niven's result was extended to arbitrary polynomial sequences, see [1], 3], [5], 8] and [10.

Throughout, we let $a$ and $b$ be positive integers. By $\left(\mathbb{Z}^{+}\right)^{\infty}$ we denote the set of all infinite sequences $\left\{s_{i}\right\}_{i=1}^{\infty}$ of positive integers (note that all the $s_{i}$ are not necessarily distinct and not necessarily monotonic). For any given $\mathcal{S}=\left\{s_{i}\right\}_{i=1}^{\infty} \in\left(\mathbb{Z}^{+}\right)^{\infty}$, we let $\mathcal{S}_{n}:=\left\{s_{1}, \ldots, s_{n}\right\}$. Associated to the infinite sequence $\mathcal{S}$ of positive integers, one can form an infinite sequence $\left\{H_{a, b}\left(\mathcal{S}_{n}\right)\right\}_{n=1}^{\infty}$ of positive rational fractions with $H_{a, b}\left(\mathcal{S}_{n}\right)$ being the

2000 Mathematics Subject Classification. primary 11N13, 11B25, 11B83, 11B75.

Key words and phrases. p-adic valuation, arithmetic progression, reciprocal power sum, Bertrand's postulate, integrality.

${ }^{*}$ Corresponding author.

$\dagger$ The research was supported partially by National Science Foundation of China Grant \#11771304 and by the Fundamental Research Funds for the Central Universities. 
$n$-th reciprocal power sum defined as follows:

$$
H_{a, b}\left(\mathcal{S}_{n}\right):=\sum_{k=1}^{n} \frac{1}{(a+(k-1) b)^{s_{k}}} .
$$

If $s_{i}=1$ for all integers $i \geq 1$, then we write $H_{a, b}(n)$ for $H_{a, b}\left(\mathcal{S}_{n}\right)$. By Nagell's theorem $[6$ and the main result of [5], we know that if $n \geq 2$ and $s_{1}=\ldots=s_{n}$, then $H_{a, b}\left(\mathcal{S}_{n}\right)$ is never an integer. In 2017, Yang, Li, Feng and Jiang [9] showed an extension of Theisinger's theorem that states that $H_{1,1}\left(\mathcal{S}_{n}\right)=1+\frac{1}{2^{s_{2}}}+\ldots+\frac{1}{n^{s_{n}}}$ is never an integer if $n \geq 2$.

In this paper, we address the problem of integrality of the $n$-th reciprocal power sum $H_{a, b}\left(\mathcal{S}_{n}\right)$. In fact, we present the following generalization of Nagell's theorem [ 6 .

Theorem 1.1. For any infinite sequence $\mathcal{S}$ of positive integers and arbitrary positive integers $a, b$, the $n$-th reciprocal power sum $H_{a, b}\left(\mathcal{S}_{n}\right)$ is never an integer if $n \geq 2$.

Letting $s_{1}=\ldots=s_{n}=1$ in Theorem 1.1 gives us Nagell's theorem [6], and picking $a=b=1$ in Theorem 1.1 yields the result of Yang, Li, Feng and Jiang 9]. The proof of Theorem 1.1 is analytic and $p$-adic in character.

This paper is organized as follows. First of all, in Section 2, we show some preliminary lemmas which are needed in the proof of Theorem 1.1. Then in Section 3, we give the proof of Theorem 1.1 .

Throughout, we always let $a, b$ and $n$ be positive integers with $n \geq 2$. As usual, for any prime $p$ and for any integer $m$, we let $v_{p}(m)$ stand for the $p$-adic valuation of $m$, i.e., $v_{p}(m)$ is the biggest nonnegative integer $r$ with $p^{r}$ dividing $m$. If $x=\frac{m_{1}}{m_{2}}$, where $m_{1}$ and $m_{2}$ are integers and $m_{2} \neq 0$, then we define $v_{p}(x):=v_{p}\left(m_{1}\right)-v_{p}\left(m_{2}\right)$.

\section{Auxiliary lemmas}

In this section, we present several auxiliary lemmas that are needed in the proof of Theorem 1.1. Clearly, $H_{a, b}\left(\mathcal{S}_{n}\right)>0$.

Lemma 2.1. Let $a=b>\frac{n}{2}$. Then $H_{a, b}\left(\mathcal{S}_{n}\right)<1$.

Proof. First, let $n=2$ or 3 . Then $a \geq 2$ since $a>\frac{n}{2}$. Hence

$$
H_{a, b}\left(\mathcal{S}_{n}\right) \leq H_{a, b}(n)=\sum_{k=1}^{n} \frac{1}{k a} \leq \sum_{k=1}^{3} \frac{1}{2 k}=\frac{11}{12}<1
$$

as expected.

Consequently, let $n=4$. Then $a \geq 3$. It follows that

$$
H_{a, b}\left(\mathcal{S}_{n}\right) \leq \sum_{k=1}^{n} \frac{1}{k a} \leq \sum_{k=1}^{4} \frac{1}{3 k}=\frac{25}{36}<1
$$

as desired.

Now let $n \geq 5$. Noting that for any positive integer $m$,

$$
(m+1) \sum_{k=1}^{m} \frac{1}{k}-m \sum_{k=1}^{m+1} \frac{1}{k}=\sum_{k=1}^{m} \frac{1}{k}-\frac{m}{m+1}>0
$$

implying that

$$
\frac{1}{m} \sum_{k=1}^{m} \frac{1}{k}>\frac{1}{m+1} \sum_{k=1}^{m+1} \frac{1}{k}
$$


one derives that

$$
H_{a, b}\left(\mathcal{S}_{n}\right) \leq \sum_{k=1}^{n} \frac{1}{k a}<\frac{2}{n} \sum_{k=1}^{n} \frac{1}{k} \leq \frac{2}{5} \sum_{k=1}^{5} \frac{1}{k}=\frac{137}{150}<1
$$

as required. So Lemma 2.1 is proved.

Lemma 2.2. Let $2 \leq n<\frac{a}{b}+1$. Then $H_{a, b}\left(\mathcal{S}_{n}\right)<1$.

Proof. First, it follows from the hypothesis that $2 \leq n<\frac{a}{b}+1$ that $a>b \geq 1$.

Firs of all, we let $a=2$. Then $b=1$ and $n=2$. So

$$
H_{a, b}\left(\mathcal{S}_{n}\right) \leq H_{2,1}(2)=\frac{1}{2}+\frac{1}{3}<1
$$

as desired.

Finally, let $a \geq 3$. Then

$$
\begin{aligned}
H_{a, b}\left(\mathcal{S}_{n}\right) & \leq \sum_{k=1}^{n} \frac{1}{a+(k-1) b} \\
& \leq \frac{1}{a}+\frac{1}{a+b}+(n-2) \frac{1}{a+2 b} \\
& <\frac{1}{a}+\frac{1}{a+b}+\left(\frac{a}{b}-1\right) \frac{1}{a+2 b} \\
& \leq \frac{1}{a}+\frac{1}{a+1}+(a-1) \frac{1}{a+2} \\
& =1-\frac{a^{2}-2 a-2}{a(a+1)(a+2)}<1
\end{aligned}
$$

as expected. Hence Lemma 2.2 is proved.

Lemma 2.3. Let $a \neq b$ and $b \in\{\operatorname{gcd}(a, b), 2 \operatorname{gcd}(a, b)\}$. If $2 \operatorname{gcd}(a, b)^{2}>a+(n-1) b$, then $H_{a, b}\left(\mathcal{S}_{n}\right)<1$.

Proof. Let $d:=\operatorname{gcd}(a, b)$. If $a=d$, then $b=2 d$ and $d>\frac{a+(n-1) b}{2 d}=\frac{2 n-1}{2}$. Hence

$$
\begin{aligned}
H_{a, b}\left(\mathcal{S}_{n}\right) & \leq H_{d, 2 d}(n) \\
& =\sum_{k=1}^{n} \frac{1}{d+2 d(k-1)} \\
& =\frac{1}{d} \sum_{k=1}^{n} \frac{1}{2 k-1} \\
& <\frac{2}{2 n-1}\left(1+\frac{n-1}{3}\right) \\
& =\frac{1}{3}+\frac{5}{6 n-3} \leq \frac{8}{9}<1
\end{aligned}
$$

as desired.

If $a \geq 2 d$, then $b \geq d$ and $d>\frac{a+(n-1) b}{2 d} \geq \frac{n+1}{2}$. Therefore

$$
H_{a, b}\left(\mathcal{S}_{n}\right) \leq \sum_{k=1}^{n} \frac{1}{2 d+d(k-1)}=\frac{1}{d} \sum_{k=1}^{n} \frac{1}{k+1}<\frac{2}{n+1} \times \frac{n}{2}<1
$$

as expected. So Lemma 2.3 is proved. 
Lemma 2.4. Let $n$ be an integer such that $2 \leq n<1+\frac{a+b}{b} e^{b\left(1-\frac{1}{a}-\frac{1}{a+b}\right)}-\frac{a}{b}$. Then $H_{a, b}\left(\mathcal{S}_{n}\right)<1$.

Proof. Since

and

$$
n-1<\frac{a+b}{b} e^{b\left(1-\frac{1}{a}-\frac{1}{a+b}\right)}-\frac{a}{b}:=c
$$

$$
\frac{1}{a+(k-1) b} \leq \int_{k-2}^{k-1} \frac{1}{a+b t} d t
$$

if $k \geq 3$, one deduces that

$$
\begin{aligned}
H_{a, b}\left(\mathcal{S}_{n}\right) & \leq H_{a, b}(n) \\
& =\sum_{k=1}^{n} \frac{1}{a+(k-1) b} \\
& \leq \frac{1}{a}+\frac{1}{a+b}+\int_{1}^{n-1} \frac{1}{a+b t} d t \\
& =\frac{1}{a}+\frac{1}{a+b}+\frac{1}{b} \log \frac{a+(n-1) b}{a+b} \\
& <\frac{1}{a}+\frac{1}{a+b}+\frac{1}{b} \log \frac{a+c b}{a+b}=1
\end{aligned}
$$

as one expects. Lemma 2.4 is proved.

Lemma 2.5. Let $a>b \geq 3$ and $(a, b) \neq(4,3)$. Then

$$
\frac{a+b}{2 b} e^{b\left(1-\frac{1}{a}-\frac{1}{a+b}\right)}-\frac{a}{2 b}+\frac{1}{2}>\frac{3 b+\sqrt{9 b^{2}+12(a-b)}}{2} .
$$

Proof. First of all, since $a>b \geq 3$ and $(a, b) \neq(4,3)$, we have $a \geq 5$ and

$$
3 b+\frac{a}{b}>\frac{3 b+\sqrt{9 b^{2}+12(a-b)}}{2} .
$$

So to prove Lemma 2.5, it suffices to show that $g(a, b)>0$ with the two-variable function $g(x, y)$ being defined by

$$
g(x, y):=\frac{x+y}{2 y} e^{y\left(1-\frac{1}{x}-\frac{1}{x+y}\right)}-3 y-\frac{3 x}{2 y}+\frac{1}{2} .
$$

Evidently, one has

$$
g(x, y)=\frac{x}{2 y}\left(e^{y\left(1-\frac{1}{x}-\frac{1}{x+y}\right)}-3\right)+\frac{1}{2} e^{y\left(1-\frac{1}{x}-\frac{1}{x+y}\right)}-3 y+\frac{1}{2}
$$

and $e^{y\left(1-\frac{1}{x}-\frac{1}{x+y}\right)} \geq e^{\frac{81}{40}} \approx 7.576110945$ when $x \geq 5$ and $y \geq 3$. Hence $g(x, y)$ increases as $x$ increases in the interval $[5, \infty)$ when $y \geq 3$.

Let $a=5$ and $b=3$. Then one can directly compute and find that

$$
\frac{a+b}{2 b} e^{b\left(1-\frac{1}{a}-\frac{1}{a+b}\right)}-\frac{a}{2 b}+\frac{1}{2} \approx 9.768147927>9.7
$$

and

$$
\frac{3 b+\sqrt{9 b^{2}+12(a-b)}}{2} \approx 9.623475385<9.7 .
$$

So Lemma 2.5 is true when $a=5$ and $b=3$. 
Let $a \geq 6$ and $b=3$. Then

$$
g(a, b)=g(a, 3) \geq g(6,3) \approx 1.59370755>0 .
$$

Finally, let $a>b \geq 4$. Then $a \geq b+1$. Noticing that $g(x, y)$ is increasing in the variable $x \in[5, \infty)$ when $y \geq 3$ and $1-\frac{1}{b+1}-\frac{1}{2 b+1} \geq \frac{31}{45}$ if $b \geq 4$, we obtain that

$$
\begin{aligned}
g(a, b) & \geq g(b+1, b) \\
& =\left(1+\frac{1}{2 b}\right) e^{b\left(1-\frac{1}{b+1}-\frac{1}{2 b+1}\right)}-3 b-\frac{3}{2 b}-1 \\
& >e^{\frac{31}{45} b}-3 b-\frac{11}{8} \\
& \geq e^{\frac{124}{45}}-12-\frac{11}{8} \\
& \approx 2.35477725>0,
\end{aligned}
$$

where the last second inequality holds due to the fact $e^{\frac{31}{45} b}-3 b-\frac{11}{8}$ increases as $b \in[4, \infty)$ increases. Hence Lemma 2.5 is proved.

Lemma 2.6. Let $b>a \geq 2$ and $4 a+b \geq 18$. Then

$$
\frac{a+b}{2 b} e^{b\left(1-\frac{1}{a}-\frac{1}{a+b}\right)}-\frac{a}{2 b}+\frac{1}{2}>3 b .
$$

Proof. First, we introduce the two-variable function $h(x, y)$ as follows:

$$
h(x, y):=\frac{x+y}{2 y} e^{y\left(1-\frac{1}{x}-\frac{1}{x+y}\right)}-\frac{x}{2 y}+\frac{1}{2}-3 y .
$$

Then

$$
h(x, y)=\frac{x}{2 y}\left(e^{y\left(1-\frac{1}{x}-\frac{1}{x+y}\right)}-1\right)+\frac{1}{2} e^{y\left(1-\frac{1}{x}-\frac{1}{x+y}\right)}+\frac{1}{2}-3 y .
$$

Hence $h(x, y)$ increases as $x$ increases in the interval $[2, \infty)$ when $y>0$. Therefore, for $b \geq 10$ and $a \geq 2$, we have

$$
\begin{aligned}
h(a, b) & \geq h(2, b) \\
& =\left(\frac{1}{b}+\frac{1}{2}\right) e^{\frac{b^{2}}{2(b+2)}}-3 b+\frac{1}{2}-\frac{1}{b} \\
& >\frac{1}{2} e^{\frac{b^{2}}{2(b+2)}}-3 b \\
& =\frac{1}{2} e^{\frac{b}{2}\left(1-\frac{2}{b+2}\right)}-3 b \\
& \geq \frac{1}{2} e^{\frac{5}{12} b}-3 b \\
& \geq \frac{1}{2} e^{\frac{25}{6}}-30 \\
& \approx 2.25004654>0 .
\end{aligned}
$$

Thus Lemma 2.6 is true when $b \geq 10$.

Now let $b \leq 9$. Then there are exactly $\left(\begin{array}{l}8 \\ 2\end{array}\right)=28$ pairs $(a, b)$ satisfying $2 \leq a<b \leq 9$ and we can calculate the values of $h(a, b)$ one by one. By some computations, we find that $h(a, b)<0$ if $(a, b)$ belongs to the following set

$$
R:=\{(2,3),(2,4),(2,5),(2,6),(2,7),(2,8),(2,9),(3,4),(3,5)\},
$$

and $h(a, b)>0$ if $(a, b)$ takes the remaining 19 pairs. In other words, $h(a, b)<0$ if $4 a+b \leq 17$, and $h(a, b)>0$ if $4 a+b \geq 18$. 
The proof of Lemma 2.6 is complete.

Lemma 2.7. Let $a \geq 2, b \geq 3$ and $a \neq b$ such that $H_{a, b}\left(\mathcal{S}_{n}\right) \geq 1$. Then there is a prime $p$ such that exactly one term in $\{a+(k-1) b\}_{k=1}^{n}$ is divisible by $p$, or $p \in\left(\max \left(\frac{n}{2}, \alpha\right), n\right]$ with

$$
\alpha:=\left\{\begin{aligned}
\frac{3 b+\sqrt{9 b^{2}+12(a-b)}}{2}, & \text { if } a>b ; \\
3 b, & \text { if } a<b .
\end{aligned}\right.
$$

Proof. Since $H_{a, b}\left(\mathcal{S}_{n}\right) \geq 1$, by Lemma 2.4. one has

$$
n \geq \frac{a+b}{b} e^{b\left(1-\frac{1}{a}-\frac{1}{a+b}\right)}-\frac{a}{b}+1 .
$$

On the other hand, by Bertrand's postulate, there is a prime $p \in\left(\frac{n}{2}, n\right]$.

If $a>b \geq 3$ and $(a, b) \neq(4,3)$, then by (4) and Lemma 2.5, one has

$$
p>\frac{n}{2} \geq \frac{a+b}{2 b} e^{b\left(1-\frac{1}{a}-\frac{1}{a+b}\right)}-\frac{a}{2 b}+\frac{1}{2}>\frac{3 b+\sqrt{9 b^{2}+12(a-b)}}{2}=\alpha
$$

as desired. So Lemma 2.7 is true in this case.

If $(a, b)=(4,3)$, then

$$
p>\frac{n}{2} \geq \frac{a+b}{2 b} e^{b\left(1-\frac{1}{a}-\frac{1}{a+b}\right)}-\frac{a}{2 b}+\frac{1}{2}=\frac{7}{6} e^{\frac{51}{28}}-\frac{1}{6} \approx 7.044128639 .
$$

But $p$ is a prime and $a=4, b=3$. So $p \geq 11>9.321825380 \approx \frac{3 b+\sqrt{9 b^{2}+12(a-b)}}{2}=\alpha$. Hence Lemma 2.7 holds in this case.

If $2 \leq a<b$ and $4 a+b \geq 18$, then by (4) and Lemma 2.6.

$$
p>\frac{n}{2} \geq \frac{a+b}{2 b} e^{b\left(1-\frac{1}{a}-\frac{1}{a+b}\right)}-\frac{a}{2 b}+\frac{1}{2}>3 b=\alpha,
$$

which means the truth of Lemma 2.7 in this case.

Now let $2 \leq a<b$ and $4 a+b \leq 17$. Then one can easily derive that the set of all the pairs $(a, b)$ equals the set $R$ given in (2). Clearly, if $n \geq 6 b$, then $p>\frac{n}{2} \geq 3 b=\alpha$, as Lemma 2.7 claimed. In what follows, we let $n \leq 6 b-1$. First, we assert that $(a, b) \notin\{(2,9),(3,5)\}$. Otherwise, one has $(a, b)=(2,9)$ or $(3,5)$. But $n \leq 6 b-1$ and a direct computation gives us that

$$
\begin{aligned}
& H_{2,9}(53)= \\
& \frac{70773412390639611995377611407286048258428112436665192898048216184826913}{72993325114428717314530010109453157943718362576137475837761483670815360}<1 \\
& \text { and } \quad H_{3,5}(29)=\frac{61763030785793910862459859011}{62877130769344946602672156032}<1 .
\end{aligned}
$$

Then it follows that for $(a, b)=(2,9)$ or $(3,5)$, we have

$$
H_{a, b}\left(\mathcal{S}_{n}\right) \leq H_{a, b}(n) \leq H_{a, b}(6 b-1)=\sum_{k=1}^{6 b-1} \frac{1}{a+(k-1) b}<1 .
$$

This contradicts with the assumption $H_{a, b}\left(\mathcal{S}_{n}\right) \geq 1$. The assertion is true.

In the following, we show that if

$$
(a, b) \in R \backslash\{(2,9),(3,5)\}=\{(2,3),(2,4),(2,5),(2,6),(2,7),(2,8),(3,4)\},
$$

then there is a prime $p$ with exactly one term in $\{a+(k-1) b\}_{k=1}^{n}$ being divisible by $p$. This will be done in what follows. 
Let $(a, b)=(2,3)$. Then

$$
H_{a, b}\left(\mathcal{S}_{n}\right) \leq H_{a, b}(n) \leq H_{2,3}(5)=\sum_{k=1}^{5} \frac{1}{3 k-1}=\frac{3041}{3080}<1
$$

if $n \leq 5$. So we must have $n \geq 6$. But $n \leq 6 b-1=17$. That is, $6 \leq n \leq 17$. We can choose $p=17=2+5 \times 3 \in\{a+(k-1) b\}_{k=1}^{n}$. So Lemma 2.7 is proved in this case.

Let $(a, b)=(2,4)$. Then

$$
H_{a, b}\left(\mathcal{S}_{n}\right) \leq H_{a, b}(n) \leq H_{2,4}(7)=\sum_{k=1}^{7} \frac{1}{4 k-2}=\frac{88069}{90090}<1
$$

if $n \leq 7$. So $n \geq 8$. But $n \leq 6 b-1=23$. Namely, $8 \leq n \leq 23$. We pick $p=13$ for $8 \leq n \leq 19$ since $2 p=2+6 \times 4 \in\{a+(k-1) b\}_{k=1}^{n}$, and $p=37$ for $20 \leq n \leq 23$ since $2 p=2+18 \times 4 \in\{a+(k-1) b\}_{k=1}^{n}$.

Let $(a, b)=(2,5)$. Then

$$
H_{a, b}\left(\mathcal{S}_{n}\right) \leq H_{a, b}(n) \leq H_{2,5}(11)=\sum_{k=1}^{11} \frac{1}{5 k-3}=\frac{3616405543}{3652567776}<1
$$

if $n \leq 11$. Thus $12 \leq n \leq 6 b-1=29$. Picking $p=47=2+9 \times 5$ gives us the desired result.

Let $(a, b)=(2,6)$. Then

$$
H_{a, b}\left(\mathcal{S}_{n}\right) \leq H_{a, b}(n) \leq H_{2,6}(17)=\sum_{k=1}^{17} \frac{1}{6 k-4}=\frac{2038704876507433}{2053923842370400}<1
$$

if $n \leq 17$. Therefore, $18 \leq n \leq 6 b-1=35$. Choosing $p=43$ gives us the required result since $2 p=2 \times 43=2+14 \times 6 \in\{a+(k-1) b\}_{k=1}^{n}$.

Let $(a, b)=(2,7)$. Then

$H_{a, b}\left(\mathcal{S}_{n}\right) \leq H_{a, b}(n) \leq H_{2,7}(27)=\sum_{k=1}^{27} \frac{1}{7 k-5}=\frac{1237220537370712858171751080193}{1241931941639876926714128796800}<1$

if $n \leq 27$. So $28 \leq n \leq 6 b-1=41$. We let $p=163=2+23 \times 7$ as one desires.

Let $(a, b)=(2,8)$. Then

$$
\begin{gathered}
H_{a, b}\left(\mathcal{S}_{n}\right) \leq H_{a, b}(n) \leq H_{2,8}(43)=\sum_{k=1}^{43} \frac{1}{8 k-6} \\
=\frac{3367642441187401373402635301280230085911262853}{3374879226092212539809802981326899789745565750}<1
\end{gathered}
$$

if $n \leq 43$. This means that $44 \leq n \leq 6 b-1=47$. Then $p=157$ leads to what we want since $2 p=2+39 \times 8 \in\{a+(k-1) b\}_{k=1}^{n}$.

Let $(a, b)=(3,4)$. Then

$$
H_{a, b}\left(\mathcal{S}_{n}\right) \leq H_{a, b}(n) \leq H_{3,4}(18)=\sum_{k=1}^{18} \frac{1}{4 k-1}=\frac{17609244113383887374}{17652709515783236895}<1
$$

if $n \leq 18$. That is, $19 \leq n \leq 6 b-1=23$. At this moment, taking $p=71=3+17 \times 4$, the desired result follows.

This finishes the proof of Lemma 2.7 
Lemma 2.8. Let $a$ and $b$ be positive integers. If $p$ is a prime and exactly one term in $\{a+(k-1) b\}_{k=1}^{n}$ is divisible by $p$, then $v_{p}\left(H_{a, b}\left(\mathcal{S}_{n}\right)\right)<0$.

Proof. Let $n_{0}$ be an integer such that $1 \leq n_{0} \leq n$ and $p \mid\left(a+\left(n_{0}-1\right) b\right)$ and $p \nmid(a+(k-1) b)$ for any integer $k$ with $k \neq n_{0}$ and $1 \leq k \leq n$. Then

$$
v_{p}\left(\frac{1}{a+\left(n_{0}-1\right) b}\right) \leq-1
$$

and

$$
v_{p}\left(\sum_{\substack{k=1 \\ k \neq n_{0}}}^{n} \frac{1}{(a+(k-1) b)^{s_{k}}}\right) \geq 0 .
$$

It follows from the isosceles triangle principle (see, for example, 4]) that

$$
\begin{gathered}
v_{p}\left(H_{a, b}\left(\mathcal{S}_{n}\right)\right)=v_{p}\left(\sum_{\substack{k=1 \\
k \neq n_{0}}}^{n} \frac{1}{(a+(k-1) b)^{s_{k}}}+\frac{1}{\left(a+\left(n_{0}-1\right) b\right)^{s_{n_{0}}}}\right) \\
=\min \left(v_{p}\left(\sum_{\substack{k=1 \\
k \neq n_{0}}}^{n} \frac{1}{(a+(k-1) b)^{s_{k}}}\right), v_{p}\left(\frac{1}{\left(a+\left(n_{0}-1\right) b\right)^{s_{n_{0}}}}\right)\right) \\
=v_{p}\left(\frac{1}{\left(a+\left(n_{0}-1\right) b\right)^{s_{n_{0}}}}\right) \leq-s_{n_{0}}<0
\end{gathered}
$$

as desired. Thus Lemma 2.8 is proved.

\section{Proof of Theorem 1.1}

We can now prove Theorem 1.1 as follows.

Proof of Theorem 1.1. Obviously, $H_{a, b}\left(\mathcal{S}_{n}\right)>0$. So we need just to prove that $H_{a, b}\left(\mathcal{S}_{n}\right)<1$ or $v_{p}\left(H_{a, b}\left(\mathcal{S}_{n}\right)\right)<0$ for some prime $p$. Let $d=\operatorname{gcd}(a, b)$. We divide the proof into the following four cases.

CASE 1. $a=b$. By Bertrand's postulate, there is a prime $p \in\left(\frac{n}{2}, n\right]$, which infers that $p \leq n<2 p$.

If $a \geq p$, then $a>\frac{n}{2}$. By Lemma 2.1. $H_{a, b}\left(\mathcal{S}_{n}\right)<1$.

If $a<p$, then $\operatorname{gcd}(a, p)=1$. Since $p \leq n<2 p$, there is only one term ap divisible by $p$ in the finite arithmetic progression $\{a k\}_{k=1}^{n}$. So by Lemma 2.8, we have $v_{p}\left(H_{a, b}\right)<0$ as desired.

CASE 2. $a \neq b$ and $b=d$ or $2 d$. Then Bertrand's postulate guarantees the existence of a prime $p \in\left(\frac{a+(n-1) b}{2 d}, \frac{a+(n-1) b}{d}\right]$.

CASE 2.1. $n<\frac{a}{b}+1$. Then $H_{a, b}\left(\mathcal{S}_{n}\right)<1$ by Lemma 2.2

CASE 2.2. $d \geq p$. Then $d \geq p>\frac{a+(n-1) b}{2 d}$. So, by Lemma $2.3, H_{a, b}\left(\mathcal{S}_{n}\right)<1$.

CASE 2.3. $n \geq \frac{a}{b}+1$ and $d<p$. Then $\frac{a+(n-1) b}{2 d} \geq \frac{a}{d}$ and $\operatorname{gcd}(d, p)=1$.

For $p \geq 3$, noticing that $\frac{b}{d}=1$ or 2 , there is exactly one term divisible by $p$ in the finite arithmetic progression $\{a+b(k-1)\}_{k=1}^{n}=\left\{d\left(\frac{a}{d}+\frac{b}{d}(k-1)\right)\right\}_{k=1}^{n}$ since $p \leq \frac{a}{d}+\frac{b}{d}(n-1)<2 p$. Immediately, Lemma 2.8 gives us that $v_{p}\left(H_{a, b}\left(\mathcal{S}_{n}\right)\right)<0$.

For $p=2$, one has $d=1$ since $d<p=2$, Since $a \neq b$ and $n \geq 2$, we have $3 \leq a+b \leq a+b(n-1)=\frac{a+b(n-1)}{d}<2 p=4$. Hence $a+b(n-1)=3$. This together with $n \geq \frac{a}{b}+1$ infers that $(n, a, b)=(2,1,2)$. That is, $H_{a, b}\left(\mathcal{S}_{n}\right)=H_{1,2}\left(\mathcal{S}_{2}\right)=1+\frac{1}{3^{s_{2}}}$, which is obviously not an integer. 
CASE 3. $a=1$ and $b \geq 3 d$. Let $H_{a, b}\left(\mathcal{S}_{n}\right)=1+H^{\prime}$, where $H^{\prime}=\sum_{k=1}^{n-1} \frac{1}{(1+k b)^{s} k+1}$. Clearly, $H^{\prime}>0$ for $n \geq 2$ and $H^{\prime}=\frac{1}{(1+b)^{s_{2}}}<1$ is not an integer if $n=2$. So it is enough to prove that either $H^{\prime}<1$ or $v_{p}\left(H^{\prime}\right)<0$ for some prime $p$ when $n \geq 3$. By Bertrand's postulate, there is a prime $p \in\left(\frac{n-1}{2}, n-1\right]$.

CASE 3.1. $n-1<\frac{1+2 b}{b} e^{b\left(1-\frac{1}{1+b}-\frac{1}{1+2 b}\right)}-\frac{1}{b}$. Then with (1) applied to $a=1$, we derive that

$$
\begin{aligned}
H^{\prime} & \leq \sum_{k=1}^{n-1} \frac{1}{1+k b} \\
& \leq \frac{1}{1+b}+\frac{1}{1+2 b}+\int_{2}^{n-1} \frac{1}{1+b t} d t \\
& =\frac{1}{1+b}+\frac{1}{1+2 b}+\frac{1}{b} \log \frac{1+b(n-1)}{1+2 b}<1
\end{aligned}
$$

as expected.

CASE 3.2. $n-1 \geq \frac{1+2 b}{b} e^{b\left(1-\frac{1}{1+b}-\frac{1}{1+2 b}\right)}-\frac{1}{b}$. Then

$$
p>\frac{n-1}{2} \geq \frac{1+2 b}{2 b} e^{b\left(1-\frac{1}{1+b}-\frac{1}{1+2 b}\right)}-\frac{1}{2 b} .
$$

We claim that $p>3 b$. Actually, if $b \geq 4$, then

$$
b\left(1-\frac{1}{1+b}-\frac{1}{1+2 b}\right) \geq b\left(1-\frac{1}{1+4}-\frac{1}{1+8}\right)=\frac{31}{45} b \geq \frac{124}{45} .
$$

But $e^{x}>\frac{135}{31} x$ for $x \geq \frac{124}{45}$. Therefore, by (5),

$$
\begin{aligned}
p & >\frac{1+2 b}{2 b} e^{\frac{31}{45} b}-\frac{1}{2 b} \\
& >\frac{1+2 b}{2 b} \times \frac{135}{31} \times \frac{31}{45} b-\frac{1}{2 b} \\
& =3 b+\frac{3 b-1}{2 b}>3 b
\end{aligned}
$$

as claimed. If $b=3$, then by (5) we have

$$
p>\frac{1+2 b}{2 b} e^{b\left(1-\frac{1}{1+b}-\frac{1}{1+2 b}\right)}-\frac{1}{2 b}=\frac{7}{6} e^{\frac{51}{28}}-\frac{1}{6} \approx 7.044128639 .
$$

But $p$ is a prime. So $p \geq 11>9=3 b$. The claim is proved.

Now from the claim, one concludes that $\operatorname{gcd}(p, b)=1$ and that there is exactly one term $1+k_{1} b$ (resp. $\left.1+k_{1} b+p b\right)$ divisible by $p$ in $\{1+k b\}_{k=1}^{p}\left(\operatorname{resp} .\{1+k b\}_{k=p+1}^{2 p}\right)$, where $1 \leq k_{1} \leq p-1$. Moreover, by the claim one has

$$
1+k_{1} b \leq 1+(p-1) b<p b<\frac{1}{3} p^{2}
$$

and

$$
1+k_{1} b+p b<2 p b<\frac{2}{3} p^{2} .
$$

Therefore, $v_{p}\left(1+k_{1} b\right)=1=v_{p}\left(1+k_{1} b+p b\right)$. Note that $1 \leq k_{1} \leq p-1 \leq n-2$ since $p \leq n-1$. In the following we show that $v_{p}\left(H^{\prime}\right)<0$. Since $p>\frac{n-1}{2}$, we have $n-1<2 p<k_{1}+2 p$. Thus there are at most two terms divisible by $p$ in $\{1+k b\}_{k=1}^{n-1}$. 
Now, if $n-1<k_{1}+p$, then there is exactly one term $1+k_{1} b$ divisible by $p$ in $\{1+k b\}_{k=1}^{n-1}=\{1+b+(k-1) b\}_{k=1}^{n-1}$. Hence by Lemma 2.8, one has

$$
v_{p}\left(H^{\prime}\right)=v_{p}\left(\sum_{k=1}^{n-1} \frac{1}{(1+k b)^{s_{k+1}}}\right)<0
$$

as expected.

If $n-1 \geq k_{1}+p$ and $s_{k_{1}+1} \neq s_{k_{1}+p+1}$, then

$$
v_{p}\left(H^{\prime}\right)=\min \left(v_{p}\left(\frac{1}{\left(1+k_{1} b\right)^{s_{k_{1}+1}}}\right), v_{p}\left(\frac{1}{\left(1+k_{1} b+p b\right)^{s_{k_{1}+p+1}}}\right)\right)<0
$$

as desired.

If $n-1 \geq k_{1}+p$ and $s_{k_{1}+1}=s_{k_{1}+p+1}:=s$, then let $H^{\prime}=A+B$, where

$$
A:=\frac{1}{\left(1+k_{1} b\right)^{s}}+\frac{1}{\left(1+k_{1} b+p b\right)^{s}}=\frac{\left(1+k_{1} b\right)^{s}+\left(1+k_{1} b+p b\right)^{s}}{\left(1+k_{1} b\right)^{s}\left(1+k_{1} b+p b\right)^{s}}
$$

and

$$
B:=\sum_{\substack{k=1 \\ k \neq k_{1}, k \neq k_{1}+p}}^{n-1} \frac{1}{(1+k b)^{s_{k+1}}} .
$$

Evidently, one has $v_{p}(B) \geq 0$. On the other hand, by (6) and (7), one has

$$
\left(1+k_{1} b\right)^{s}+\left(1+k_{1} b+p b\right)^{s}<\left(\frac{1}{3} p^{2}\right)^{s}+\left(\frac{2}{3} p^{2}\right)^{s} \leq p^{2 s}
$$

and so $v_{p}\left(\left(1+k_{1} b\right)^{s}+\left(1+k_{1} b+p b\right)^{s}\right)<2 s$. But $v_{p}\left(\left(1+k_{1} b\right)^{s}\left(1+k_{1} b+p b\right)^{s}\right)=2 s$. Therefore we have

$$
v_{p}(A)=v_{p}\left(\left(1+k_{1} b\right)^{s}+\left(1+k_{1} b+p b\right)^{s}\right)-v_{p}\left(\left(1+k_{1} b\right)^{s}\left(1+k_{1} b+p b\right)^{s}\right)<0
$$

and

$$
v_{p}\left(H^{\prime}\right)=v_{p}(A+B)=\min \left(v_{p}(A), v_{p}(B)\right)=v_{p}(A)<0
$$

as required. So Theorem 1.1 is proved in this case.

CASE 4. $a \neq b, a \geq 2$ and $b \geq 3 d$. We revise the argument of Case 3. Clearly, we just need to deal with the case when $H_{a, b}\left(\mathcal{S}_{n}\right) \geq 1$. In what follows, let $H_{a, b}\left(\mathcal{S}_{n}\right) \geq 1$. Then by Lemma 2.7 there is a prime $p$ such that exactly one term in $\{a+(k-1) b\}_{k=1}^{n}$ is divisible by $p$, or $p \in\left(\max \left(\frac{n}{2}, \alpha\right), n\right]$ with $\alpha$ being given in (3).

If there is a prime $p$ such that exactly one term in $\{a+(k-1) b\}_{k=1}^{n}$ is divisible by $p$, then by Lemma 2.8, we have $v_{p}\left(H_{a, b}\left(\mathcal{S}_{n}\right)\right)<0$. If it doesn't hold, then by Lemma 2.7, there is a prime $p \in\left(\max \left(\frac{n}{2}, \alpha\right), n\right]$. Since $p>\alpha \geq 3 b$, we have $\operatorname{gcd}(p, b)=1$ and in $\{a+k b\}_{k=0}^{p-1}$ (resp. $\{a+k b\}_{k=p}^{2 p-1}$ ), there is exactly one term $a+k_{2} b$ (resp. $a+k_{2} b+p b$ ) divisible by $p$, where $0 \leq k_{2} \leq p-1$. But $p-1 \leq n-1<2 p-1$, it implies that there are exactly two terms $a+k_{2} b$ and $a+k_{2} b+p b$ in $\{a+(k-1) b\}_{k=1}^{n}$ divisible by $p$. Since $0 \leq k_{2} \leq p-1$, we deduce that $a+k_{2} b \leq p b+a-b$. If $a>b$, then $p>\alpha=\frac{3 b+\sqrt{9 b^{2}+12(a-b)}}{2}$ from which one derives that

$$
p b+a-b<\frac{1}{3} p^{2} .
$$

If $a<b$, then $a-b<0$ and $p>\alpha=3 b$, and so (8) still holds when $a<b$. Hence

$$
p \leq a+k_{2} b \leq p b+a-b<\frac{1}{3} p^{2} .
$$


This infers that $v_{p}\left(a+k_{2} b\right)=1$. But $p>\alpha \geq 3 b$. Thus $p b<\frac{1}{3} p^{2}$ and by (9),

$$
p \leq a+k_{2} b+p b<\frac{2}{3} p^{2} .
$$

It follows that $v_{p}\left(a+k_{2} b+p b\right)=1$.

First, let $s_{k_{2}+1} \neq s_{k_{2}+p+1}$. Then

$$
\begin{aligned}
v_{p}\left(H_{a, b}\left(\mathcal{S}_{n}\right)\right)=\min & \left(v_{p}\left(\frac{1}{\left(a+k_{2} b\right)^{s_{k_{2}+1}}}\right), v_{p}\left(\frac{1}{\left(a+k_{2} b+p b\right)^{s_{k_{2}+p+1}}}\right)\right) \\
= & \min \left(-s_{k_{2}+1},-s_{k_{2}+p+1}\right)<0
\end{aligned}
$$

as required.

Now let $s_{k_{2}+1}=s_{k_{2}+p+1}:=\bar{s}$. Then we split $H_{a, b}\left(\mathcal{S}_{n}\right)$ into two parts: $H_{a, b}\left(\mathcal{S}_{n}\right)=$ $C+D$, where

$$
C:=\frac{1}{\left(a+k_{2} b\right)^{\bar{s}}}+\frac{1}{\left(a+k_{2} b+p b\right)^{\bar{s}}}=\frac{\left(a+k_{2} b\right)^{\bar{s}}+\left(a+k_{2} b+p b\right)^{\bar{s}}}{\left(a+k_{2} b\right)^{\bar{s}}\left(a+k_{2} b+p b\right)^{\bar{s}}}
$$

and

$$
D:=\sum_{\substack{k=1 \\ k \neq k_{2}+1, k \neq k_{2}+p+1}}^{n} \frac{1}{(a+(k-1) b)^{s_{k}}} .
$$

However, from (8) and (9), one deduces that

$$
0<\left(a+k_{2} b\right)^{\bar{s}}+\left(a+k_{2} b+p b\right)^{\bar{s}}<\left(\frac{1}{3} p^{2}\right)^{\bar{s}}+\left(\frac{2}{3} p^{2}\right)^{\bar{s}} \leq p^{2 \bar{s}} .
$$

So $v_{p}\left(\left(a+k_{2} b\right)^{\bar{s}}+\left(a+k_{2} b+p b\right)^{\bar{s}}\right)<2 \bar{s}$. But $v_{p}\left(\left(a+k_{2} b\right)^{\bar{s}}\left(a+k_{2} b+p b\right)^{\bar{s}}\right)=2 \bar{s}$. Thus

$$
v_{p}(C)=v_{p}\left(\left(a+k_{2} b\right)^{\bar{s}}+\left(a+k_{2} b+p b\right)^{\bar{s}}\right)-v_{p}\left(\left(a+k_{2} b\right)^{\bar{s}}\left(a+k_{2} b+p b\right)^{\bar{s}}\right)<0 .
$$

Since $v_{p}(D) \geq 0$, we deduce immediately that

$$
v_{p}\left(H_{a, b}\left(\mathcal{S}_{n}\right)\right)=v_{p}(C+D)=v_{p}(C)<0
$$

as one desires.

This concludes the proof of Theorem 1.1

\section{Final Remarks}

Let $a, b$ and $n$ be positive integers. For any integer $k$ with $1 \leq k \leq n, H_{a, b}^{(k)}\left(\mathcal{S}_{n}\right)$ stands for the $k$-th elementary symmetric function of the $n$ fractions: $\frac{1}{a^{s_{1}}}, \frac{1}{(a+b)^{s_{2}}}, \ldots, \frac{1}{(a+(n-1) b)^{s_{n}}}$. Namely,

$$
H_{a, b}^{(k)}\left(\mathcal{S}_{n}\right):=\sum_{1 \leq i_{1}<\ldots<i_{k} \leq n} \prod_{j=1}^{k} \frac{1}{\left(a+b\left(i_{j}-1\right)\right)^{s_{i_{j}}}} .
$$

Then $H_{a, b}^{(1)}\left(\mathcal{S}_{n}\right)=H_{a, b}\left(\mathcal{S}_{n}\right)$. Hong and Wang 3] showed that if all $s_{i}$ are equal to 1 , then all $H_{a, b}^{(k)}\left(\mathcal{S}_{n}\right)$ are not integers if $n \geq 4$. We believe that such result is still true for any infinite sequence $\mathcal{S}$ of positive integers. Namely, we propose the following conjecture.

Conjecture 4.1. For any infinite sequence $\mathcal{S}$ of positive integers and arbitrary positive integers $a, b$ and $n$, if $n \geq 4$, then none of $H_{a, b}^{(1)}\left(\mathcal{S}_{n}\right), H_{a, b}^{(2)}\left(\mathcal{S}_{n}\right), \ldots, H_{a, b}^{(n)}\left(\mathcal{S}_{n}\right)$ is an integer.

By Theorem 1.1, one knows that Conjecture 4.1 is true when $k=1$. It is clear that Conjecture 4.1 holds when $k=n$. Hence we need just to look at the case $2 \leq k \leq n-1$. 
On the other hand, if all $s_{i}$ are equal to 1, then Hong and Wang's result [3] says that Conjecture 4.1 is true. If all $s_{i}$ are greater than 2 , then one can show the truth of Conjecture 4.1. However, if there exist indexes $i$ and $j$ such that $s_{i}=1$ and $s_{j} \geq 2$, then the situation becomes complicated and hard, and so the truth of Conjecture 4.1 is still kept open so far.

\section{ACKNOWLEDGEMENT}

The authors would like to thank the anonymous referee for careful reading of the manuscript and helpful comments.

\section{REFERENCES}

1. Y.G. Chen and M. Tang, On the elementary symmetric functions of $1,1 / 2, \ldots, 1 / n$, Amer. Math. Monthly, 119 (2012), 862-867.

2. P. Erdös and I. Niven, Some properties of partial sums of the harmonic series, Bull. Amer. Math Soc., 52 (1946), 248-251.

3. S.F. Hong and C.L. Wang, The elementary symmetric functions of reciprocals of the elements of arithmetic progressions, Acta Math. Hungari., 144 (2014), 196-211.

4. N. Koblitz, $p$-Adic numbers, $p$-adic analysis and zeta-functions, 2nd ed., Springer-Verlag (New York, 1984).

5. Y.Y. Luo, S.F. Hong, G.Y. Qian and C.L. Wang, The elementary symmetric functions of a reciprocal polynomial sequence, C.R. Acad. Sci. Paris, Ser. I, 352 (2014), 269-272.

6. T. Nagell, Eine Eigenschaft gewissen Summen, Skr. Norske Vid. Akad. Kristiania, 13 (1923), 10-15.

7. L. Theisinger, Bemerkung über die harmonische Reihe, Monatsh. Math. Phys., 26 (1915), 132-134.

8. C.L. Wang and S.F. Hong, On the integrality of the elementary symmetric functions of $1,1 / 3, \ldots$, 1/(2n-1), Math. Slovaca, 65 (2015), 957-962.

9. W.X. Yang, M. Li, Y.L. Feng and X. Jiang, On the integrality of the first and second elementary symmetric functions of $1,1 / 2^{s_{2}}, \ldots, 1 / n^{s_{n}}$, AIMS Math., 2 (2017), 682-691.

10. Q.Y. Yin, S.F. Hong, L.P. Yang and M. Qiu, Multiple reciprocal sums and multiple reciprocal star sums of polynomials are almost never integers, J. Number Theory, https://doi.org/10.1016/j.jnt.2018.06.005, published online July 17, 2018. 